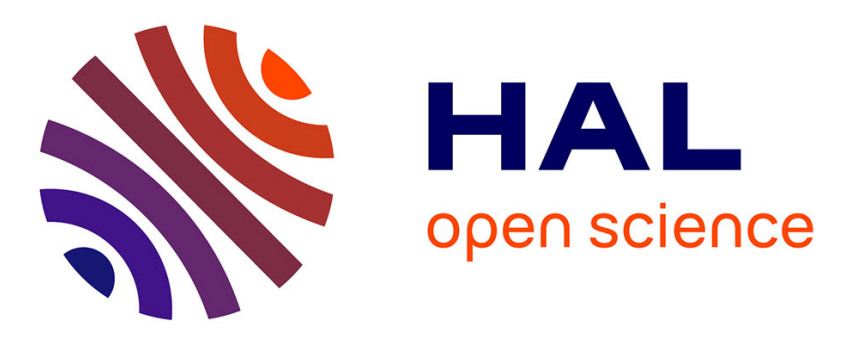

\title{
Experimental clues of soft glassy rheology in strained filled elastomers
}

Françoise Ehrburger-Dolle, Isabelle Morfin, Françoise Bley, Frédéric Livet, Gert Heinrich, Luc Piché, Mark Sutton

\section{- To cite this version:}

Françoise Ehrburger-Dolle, Isabelle Morfin, Françoise Bley, Frédéric Livet, Gert Heinrich, et al.. Experimental clues of soft glassy rheology in strained filled elastomers. Journal of Polymer Science Part B: Polymer Physics, 2014, 52, pp.647-656. 10.1002/POLB.23463 . hal-01006630

\section{HAL Id: hal-01006630 \\ https://hal.science/hal-01006630}

Submitted on 16 Jun 2014

HAL is a multi-disciplinary open access archive for the deposit and dissemination of scientific research documents, whether they are published or not. The documents may come from teaching and research institutions in France or abroad, or from public or private research centers.
L'archive ouverte pluridisciplinaire HAL, est destinée au dépôt et à la diffusion de documents scientifiques de niveau recherche, publiés ou non, émanant des établissements d'enseignement et de recherche français ou étrangers, des laboratoires publics ou privés. 


\title{
Experimental Clues of Soft Glassy Rheology in Strained Filled
}

\section{Elastomers}

\section{Françoise Ehrburger-Dolle ${ }^{1}$, Isabelle Morfin ${ }^{1}$, Françoise Bley ${ }^{2}$, Frédéric Livet ${ }^{2}$, Gert}

Heinrich $^{3}$, Luc Piché ${ }^{4}$, Mark Sutton ${ }^{4}$

${ }^{1}$ CNRS/Univ. Grenoble 1, Laboratoire Interdisciplinaire de Physique, UMR 5588, Grenoble, F38041, France

${ }^{2}$ CNRS/Grenoble INP/Univ. Grenoble 1, SIMaP, UMR 5266, 38402 Saint Martin d'Hères,

France

${ }^{3}$ Leibniz-Institut für Polymerforschung Dresden e.V., 010169 Dresden, Germany

${ }^{4}$ Physics Department, McGill University, Montreal, Quebec H3A 2T8, Canada

Correspondence to: F. Ehrburger-Dolle (Email: francoise.ehrburger-dolle@ujf-grenoble.fr)

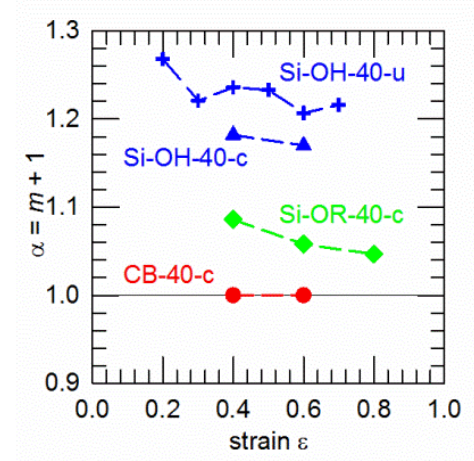

For up jump steps, the tensile relaxation modulus $E(t)$ decreases as $\log (t)$ for carbon black filled samples and as $E(t)=E_{\infty}\left[1+\left(t / \tau_{0}\right)^{-m}\right]$ for the silica ones. Thus the rate of change of $E(t),|d E(t) / d t|$, scales as $t^{-\alpha}$ where $\alpha=m+1$. The equivalence of $\alpha$ (empirical) and $x$ (effective noise temperature in the SGR model) which would be related to the polymer chain mobility at a given strain $\varepsilon$, is discussed.

\begin{abstract}
Tensile stress-relaxation measurements have been performed on a series of cross-linked filled elastomers. The fillers are chosen in order to investigate the effect of the filler-filler and the filler-matrix interactions on the time dependence of the tensile relaxation modulus $E(t)$ after UP and DOWN jumps. For the carbon black filled sample (strong filler-elastomer interaction) $E(t)$ decreases as $\log (t)$ when the strain $\varepsilon$ is strictly larger than 0.2 and reached by UP jumps. For the
\end{abstract}


silica filled samples in the same conditions, and for all samples after a DOWN jump, including $\varepsilon=0.2$, the experimental data can be fitted with a power law equation characterized by the exponent $m$. Thus, in all cases, $|d E(t) / d t|$ scales as $t^{-\alpha}$ with $\alpha=m+1$. Pertinence of the Soft Glassy Rheology (SGR) model for interpreting the present results is examined. It is shown that $\alpha$ could be equivalent to the effective noise temperature $x$ and related to the polymer chain mobility.

KEY WORDS: filled elastomers; tensile stress relaxation; soft glassy rheology

\section{INTRODUCTION}

The mechanical behavior of filled elastomers is an active research area since more than 60 years mainly because it is related to an ancient and important application which is the reinforcement of rubber. The large number of recent reviews [1-7] related to this subject reveals its complexity. The internal structure of nanocomposites in general and of filled elastomers, in particular, leads to complicated time dependent deformations in response to a mechanical strain. Therefore the relation between stress and strain cannot be simply described by elastic and viscous characteristics because they are intricate function of time and extent of strain [8]. The study of the response of a viscoelastic material to step or oscillatory strains or to a constant stress (creep strain) is one of the most straightforward methods for getting information about the contribution of time and strain separately. Early measurements of tensile stress relaxation $\sigma(t)$ in a series of carbon black N330 filled rubber vulcanizates were reported by MacKenzie and Scalan [9]. A linear regression of the experimental data plotted in a semi-logarithmic system of axes indicated a logarithmic decrease of the stress $\sigma(t)=A-B \log (t)$. Fully logarithmic plots allowed also a linear regression of the data suggesting a power law decrease of the strain $\sigma(t)=a t^{-n}$ for $t$ larger than about $60 \mathrm{~s}$. In all cases the exponents were smaller than 0.04 . It follows that the 
logarithmic or the power law fits are nearly equivalent. Logarithmic stress relaxations were also reported in several vulcanized rubbers unfilled or filled with carbon black $[10,11]$. Actually, tens of references dealing with stress relaxation in filled or unfilled elastomers can be found in the literature. As a result of the lack of models, representation of the experimental data in semilogarithmic or logarithmic coordinates was essentially aiming to compare qualitatively the mechanical responses of different systems [12]. Cross-linked rubbers exhibit a finite stress $\sigma_{\infty}$ in the long-time limit in contrast to uncross-linked rubbers with no equilibrium stress $\left(\sigma_{\infty}=0\right)$. It follows that, in the latter case, a power law relaxation appears as a straight line when experimental data are plotted in logarithmic coordinates over several decades in time. Such a result was also reported by Mani and coll. [13] for an interpenetrating polymer network of polyacrylate and polystyrene. In contrast, for cross-linked rubbers or any other system exhibiting a long-time non-zero stress, a power law relaxation, if any, would be observed by plotting $\Delta \sigma(t)=\sigma(t)-\sigma_{\infty}$ in logarithmic coordinates. Because $\sigma_{\infty}$ is generally unknown, it is easier to examine the quality of a non-linear regression of the data by means of the following equation: $\sigma(t)=a t^{-m}+\sigma_{\infty}$. In fact, this equation is similar to the one proposed, more than 50 years ago, by Chasset and Thirion [14] for fitting the isothermal stress relaxation modulus of natural rubber cross-linked with dicumyl peroxide:

$E(t)=E_{\infty}\left[1+\left(t / \tau_{0}\right)^{-m}\right]$

In this equation, $E_{\infty}$ is the long time equilibrium modulus, $\tau_{0}$ is a characteristic time and $m$ is the power law exponent. The same type of equation applies also to the shear modulus relaxation $G(t)$ or to any other stress or strain relaxation occurring in cross-linked systems. The ChassetThirion (CT) equation has been widely used to describe power law stress relaxation in unfilled cross-linked polymers or elastomers [15-21]. Values of $m$ ranging between 0.1 and 0.3 are 
generally reported. More recently, Mitra and coll. [22] have shown that the CT equation fitted well the relaxation modulus of cross-linked nanogel filled elastomers. The above examples reveal power law relaxation in un-filled and filled cross-linked systems. As a matter of fact, there are many classes of soft materials, including living cells [23-28] in which stress relaxation following a step strain are well described by a power law decrease in time $\left(G(t)\right.$ or $\left.E(t) \propto t^{-\beta}\right)$ or an equation similar to the CT one [28-31].

There have been several attempts [32-34] to model the empirical power law equation describing the stress relaxation in cross-linked polymers proposed by Chasset and Thirion. These models were based on the assumption that relaxation is due to dangling chains but the theories differed in the prediction of the relation between the cross-linking density and the exponent $m$ [35]. More generally, a power law relaxation implies a broad distribution of relaxation times which is typical of structural disorder and metastability as it is the case for soft glasses also named "soft glassy materials" (SGM) [36-38]. In order to rationalize the rheology of this class of materials, Sollich [37] proposed the "soft glassy rheology" (SGR) model. In this model, each individual element is located within an energy landscape containing many wells of different depth from which the elements are unlikely to escape by thermal fluctuations alone. The elements are trapped in cages formed by their neighbors. Under the effect of an activation energy, for example a strain, these elements (corresponding to mesoscopic regions) may hop out of a trap. It is expected that these "activated" yield processes arise primarily by coupling to structural rearrangements elsewhere in the system. In a mean-field spirit, all such interactions between regions are subsumed into an effective "noise temperature" $x$ [36]. $x$ is related to the power law exponent by the relation: $\beta=x-1$. In the case of viscoelastic systems, $x$ is comprised between 1 and 2 . When $x$ tends toward unity, the elements are trapped into deeper and deeper wells and the system approaches a 
glass transition. More recently, Bouchbinder and Langer [39] on the one hand, Fuereder and Ilg [40], on the other hand, have addressed the question of the issue of a proper thermodynamic interpretation of SGR. These authors show that the effective temperature $x$ as it appears in the SGR model should be identical to the configurational temperature $\chi$ associated with the slow degree of freedom.

Coming back to cross-linked elastomers (un-filled or filled), it is now interesting to raise the following questions. Is it pertinent to consider them as soft glassy materials? The possibility of a link between the physics of colloidal glasses and that of filled rubbers has been recently evoked $[41,42]$. If so, is it pertinent to relate the power law exponent $m$ to the effective noise temperature $x$ by means of $m=x-1$ ? Examination of these questions is essential as positive answers would bring new insights in the physics of strained filled elastomers. The aim of the present work is to search for experimental clues by means of the analysis of the behavior of the tensile relaxation modulus $E(t)$ at a given strain reached by a strain step $\Delta \varepsilon=\varepsilon_{f}-\varepsilon_{i}$ where $\varepsilon_{i}$ and $\varepsilon_{f}$ are the initial and the final values of the strain respectively. In order to investigate sequences effects [43] known as Mullins effect in filled elastomers [44, 45], positive (up-jump experiment) and negative (down-jump experiment) steps will be considered. Because the fillerfiller and the filler-matrix interactions play an essential role in the properties and the dynamics of filled rubbers [46-49] and more generally, of polymer nanocomposites [50, 51], different nanoparticles (hydrophilic or hydrophobic silica, carbon black) were used as fillers in an Ethylene Propylene Diene Monomer (EPDM) cross-linked rubber. The influence of cross-linking was approached by comparing the results obtained for the hydrophilic silica filled elastomer cross-linked and uncross-linked. 


\section{MATERIALS AND EXPERIMENTAL METHODS}

\section{Samples}

The elastomer matrix investigated is an Ethylene Propylene Diene Monomer (EPDM) rubber (Buna EP G 6850, Lanxess, Leverkusen, Germany). Three different fillers were used: carbon black N330 (Evonik Carbon Black GmbH), hydroxylated pyrogenic silica AEROSIL® 200 (Degussa) and AEROSIL® R 974. The latter is a hydrophobic fumed silica aftertreated with dimethyldichlorosilane based on the hydrophilic AEROSIL $® 200$ with a specific surface area

close to $200 \mathrm{~m}^{2} / \mathrm{g}$. The morphological characteristics of the fillers have been previously described in details [52-54]. The filled elastomers were prepared by mixing $40 \mathrm{~g}$ of filler with $100 \mathrm{~g}$ of rubber (i.e., $40 \mathrm{phr}$ ) and $3 \mathrm{~g}$ of dicumyl peroxide (the cross-linking agent) in a two-roll mill for 5 minutes at $50^{\circ} \mathrm{C}$. Afterwards, the mixtures were cured in molds at $160^{\circ} \mathrm{C}$ under pressure during 10 minutes. The volume fraction of filler $\phi$ corresponding to $40 \mathrm{phr}$ is close to 0.16 for the carbon black filled sample and to 0.14 for the silica ones. In both cases, the volume fraction is expected to be above the "percolation" threshold which is close to 0.10 for carbon black N330 [55] and possibly smaller for AEROSIL® 200 [56, 57]. However, because the specific surface area of the two silica fillers is larger than that of the carbon black one, the extent of the filler matrix interface is larger for the formers than for the latter. In the following, the names Si-OH-40-c, Si-OR-40-c and CB-40-c will refer to cross-linked samples with 40 phr of AEROSIL® 200, AEROSIL® R 974 and carbon black, respectively. The uncross-linked AEROSIL ${ }^{\circledR} 200$ filled sample will be named Si-OH-40-u. The thickness $e$ of the plates was equal to $1 \mathrm{~mm}$. For all measurements, the plates were punched out to the classical dumb-bell shape. The width $l$ of the gauge was equal to $4 \mathrm{~mm}$ and its length was equal to $25 \mathrm{~mm}$. 


\section{Tensile force measurements}

Tensile relaxation measurements performed at given steps of strain (incremental stress-strain curves) were achieved by means of the apparatus designed for simultaneous stress and Heterodyne X-Ray Photon Correlation Spectroscopy (HD-XPCS) study [58]. A complete description can be found in the master thesis of L.J.S. Halloran [59]. Each strain step was reached after a rapid extension $\left(0.034 \mathrm{~s}^{-1}\right)$ from the previous step to the next one. A strain gauge and a digitizing unit were used to measure the evolution of the force $F$ every 1 or $2 \mathrm{~s}$ at each elongation step during about $3000 \mathrm{~s}$. The value of the stress $\sigma$ is deduced from $F$ by $\sigma=F / A$ where $A=e l$ is the initial section of the sample $\left(A=4 \mathrm{~mm}^{2}\right)$. The stretch ratio $\lambda$ is defined as $\lambda=L / L_{0}$ where $L$ and $L_{0}$ are the final and the initial lengths respectively. The strain $\varepsilon$ is defined as $\varepsilon=\left(L-L_{0}\right) / L_{0}=\lambda-1$ (engineering strain). The tensile relaxation modulus $E(t)$ is defined as $E(t)=\sigma(t) / \varepsilon$. Fitting of the experimental curves $E(t)$ is achieved by non-linear regression procedures by means of the Marquardt - Levenberg algorithm provided by SigmaPlot 10.0.

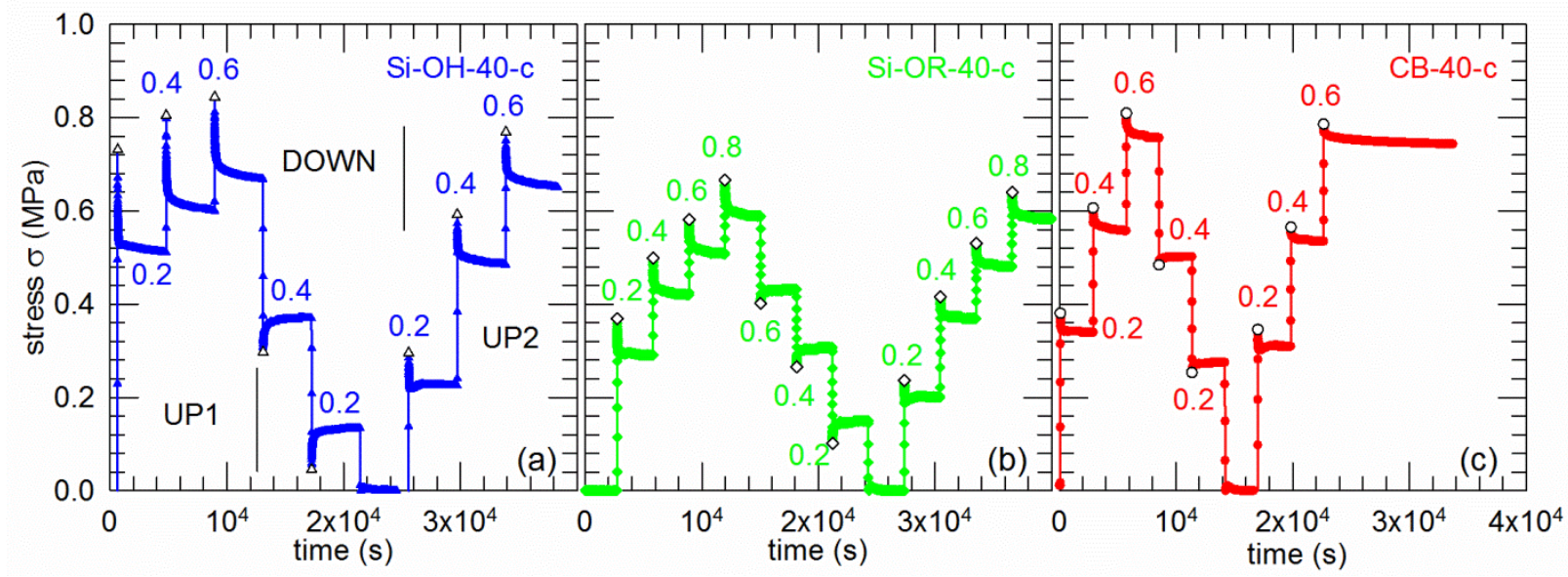

FIGURE $1(\mathbf{a}, \mathbf{b}, \mathbf{c})$ Incremental stress-strain curves. The open black symbols indicate the origin $(t=0)$ of the time scale in the relaxation curves. The numbers near the curves indicate the value of the strain $\varepsilon$. 
The curves $\sigma(t)=F(t) / A$ obtained for the different samples are shown in Figure 1. In all cases, the difference between each strain step is equal to 0.2 . The maximum strain is 0.6 for $\mathrm{Si}$ OH-40-c and CB-40-c and 0.8 for Si-OR-40-c. The black open symbols shown in the figures indicate the origin of the time scale $(t=0)$ for the tensile relaxation analysis. This figure shows that the amplitude of the stress change at each strain plateau decreases when going from hydroxylated to hydrophobic silica and to the carbon black filled sample. For the uncross-linked sample Si-OH-40-u, the stress relaxation modulus was measured for strain steps varying between 0.1 and 0.7 . For this sample, the jump between each step was 0.1 and the sequence was limited to the UP1 curves.

\section{ANALYSIS OF THE EXPERIMENTAL DATA}

\section{Experimental results obtained for $\varepsilon=0.4$}

The time dependence of the tensile relaxation modulus $E(t)=\sigma(t) / \varepsilon$ obtained for the 3 samples for $\varepsilon=0.4$ is plotted in Figures 2a, b, c. For both silica filled samples, the UP1 and UP2 data (for $t \geq 1 \mathrm{~s}$ ) are well fitted by eq 1 as it was reported for unfilled cross-linked rubbers [1521] and for nanogels filled elastomers [22]. The parameters deduced from the fits and their standard errors are collected in Table 1. After the first UP-jump (UP1), the value of the exponent $m$ is significantly larger in the case of the hydroxylated silica than for the hydrophobic one. After the second UP-jump (UP2), the value of $m$ obtained for Si-OH-40-c (0.11) is slightly smaller than the one obtained for Si-OR-40-c (0.12). However, it must be reminded that, as shown in Figure 1, the mechanical history is not exactly the same: sample Si-OH-40-c was strained up to 0.6 while Si-OR-40-c was strained up to 0.8 . The values of the equilibrium tensile modulus $E_{\infty}$ deduced from the fits will be discussed in the last part of the paper. The order of magnitude of 
the characteristic times $\tau_{0}$ (Table 1 ) is the same as that of the $\tau_{0}$ values reported for some crosslinked elastomers. In the present paper, $\tau_{0}$ will be considered as a time scaling factor but it will not be further discussed.

For the carbon black filled sample (Figure 2c), the data obtained after the first and the second jump (UP1 and UP2) display a linear relation between $E(t)$ and $\log (t)$. It follows that, as it has been earlier observed for carbon black filled elastomers [9], the tensile relaxation modulus displays a logarithmic decrease:

$$
E(t)=E(1)[1-K \log (t)]
$$

The values of $K$ (figure $2 \mathrm{c}$ ) are very small. It follows that the introduction of a characteristic time $\tau_{0}$ at which the tensile modulus would be equal to zero is un-physically large.

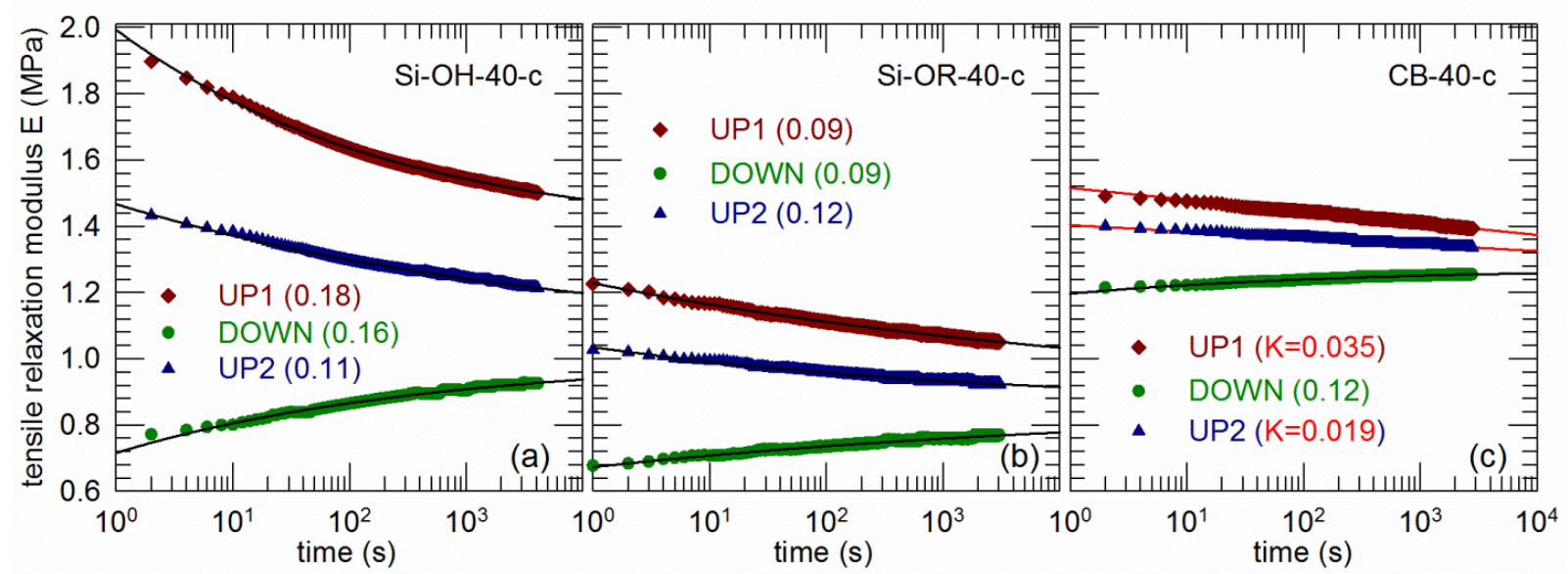

FIGURE 2 Semi-logarithmic plots of the time dependence of the tensile relaxation modulus $E$ for $\varepsilon=0.4$ : (a) Si-OH-40-c, (b) Si-OR-40-c and (c) CB-40-c samples; solid lines are fits with eq 1 [the values of the power law exponent $m$ are indicated in brackets]; dashed red lines in (c) are fits with eq 2 
Figure 2 shows that after a down-jump from $\varepsilon=0.6$ to 0.4 , the tensile modulus increases with time. The experimental data, including that obtained for the carbon black filled sample, can be properly fitted by means of a power law equation which is similar to eq 1 :

$$
E^{\#}(t)=E_{\infty}\left[1-\left(t / \tau_{0}\right)^{-m}\right] \quad \text { with } m>0
$$

This empirical observation allows us to introduce an alternative equation able to describe both UP and DOWN jumps:

$$
E(t)=E_{\infty}\left[1+k\left(t / \tau_{0}\right)^{-m}\right]
$$

in which: $\quad k=1$ for positive strain steps ("UP", $\Delta \varepsilon>0$ )

$k=-1$ for negative strain steps ("DOWN", $\Delta \varepsilon<0$ ).

\begin{tabular}{ll|cccc}
\multicolumn{2}{c|}{$\varepsilon=0.4$} & $\mathrm{E}(0)(\mathrm{MPa})$ & $\mathrm{E}_{\infty}(\mathrm{MPa})$ & $m$ & $\tau_{0}(\mathrm{~s})$ \\
\hline \multirow{2}{*}{ Si-OH-40-c } & UP1 & 2.010 & $1.364 \pm 0.001$ & $0.182 \pm 0.001$ & $0.0143 \pm 0.0002$ \\
& UPWN & 0.744 & $1.000 \pm 0.002$ & $0.163 \pm 0.003$ & $(4.5 \pm 0.6) \times 10^{-4}$ \\
& UP2 & 1.480 & $1.047 \pm 0.004$ & $0.111 \pm 0.002$ & $(2.7 \pm 0.2) \times 10^{-4}$ \\
\hline \multirow{2}{*}{ Si-OR-40-c } & DOWN & 1.246 & $0.871 \pm 0.004$ & $0.086 \pm 0.002$ & $(3.1 \pm 0.1) \times 10^{-5}$ \\
& UP2 & 1.038 & $0.856 \pm 0.003$ & $0.122 \pm 0.004$ & $(2.7 \pm 0.8) \times 10^{-6}$ \\
\hline CB-40-c & DOWN & 1.209 & $1.273 \pm 0.001$ & $0.117 \pm 0.004$ & $(1.3 \pm 0.4) \times 10^{-7}$ \\
\hline
\end{tabular}

TABLE 1 Parameters deduced from the fit of the data shown in Figure 2 by eq 1 ; the sign \# indicates $k=-1$ in eq 4

Figure 2 deserves two more comments. Firstly, the significant difference between the relaxation curves measured after the first step (UP1) and the second one (UP2) reveals the hysteresis in the 
stress-strain curves expected in the case of filled elastomers (Mullins effect $[44,45]$ ) as in unfilled cross-linked rubbers [3]. The second comment concerns the asymmetry observed in the UP and DOWN jump curves also observed in cross-linked natural rubber [3]. These features are similar to the ones observed in asymmetry of approach experiments (temperature jumps) in polymer glasses [43]. Wang and Robertson [41] reported the same asymmetry effect for the storage modulus $G^{\prime}$ for carbon black filled polybutadiene rubbers. These authors also noted that this asymmetric behavior cannot be described by the kinetic models proposed in the literature [60-62]. In the present case, experimental facts allows us to show that the same power law equation is able to describe the relaxation after UP and DOWN jumps, but the parameters $m, \tau_{0}$ and $E_{\infty}$ are different. Interestingly, as shown in Table 1, the asymptotic values $E_{\infty}(\mathrm{DOWN})$ are very close from $E_{\infty}(\mathrm{UP} 2)$ for the two silica filled samples.

\section{Experimental results obtained for $\varepsilon=0.6$ and 0.8}

The experimental results obtained for $\varepsilon=0.6$ are plotted in Figure 3. In all cases, the difference between the UP1 and UP2 curves is less pronouced than it was for $\varepsilon=0.4$ (Figure 2) but the trends are the same. The asymmetry of approach is observed for the hydrophobic silica sample Si-OR-40-c which had been stretched at 0.8 . For all silica filled samples, the relaxation is well described by the power law equation (eq 4), but the values of the fitting parameters are different. Particularly, the value of the exponent $m$ and that of the asymptotic limit becomes smaller (these questions will be discussed in the next part). For the carbon black filled sample CB-40-c, the UP jumps yield a logarithmic decrease of the relaxation modulus while, as in the previous case, the DOWN-jump recovery follows the power-law equation (eq 4). As for the time dependence of the tensile relaxation modulus for $\varepsilon=0.8$ (not shown) measured for Si-OR-c, the amplitude of the 
diminution of $E(t)$ within the time window considered becomes quite small and the exponent $m$ is close to 0.05 for both UP1 and UP2 curves.
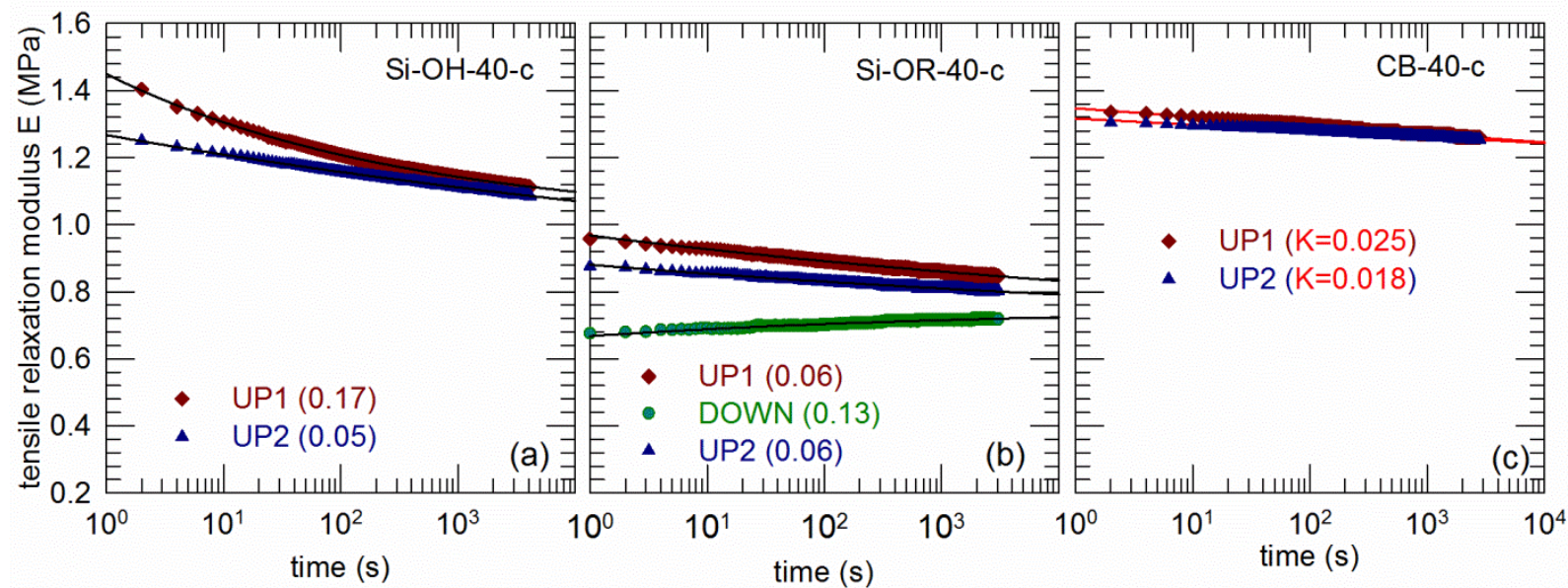

FIGURE 3 Time dependence of the tensile relaxation modulus $\mathrm{E}$ for $\varepsilon=0.6$; the values of $m$ are given in brackets

\section{Experimental results obtained for $\varepsilon=0.2$}

The shape of the curves plotted in Figure 4 for $\varepsilon=0.2$ (UP1 and UP2) is quite different from the previous ones measured at a larger strain. The DOWN curves are similar to the previous ones. We have verified that the UP1 curves cannot be fitted by means of the power law equation (eq 4). Stretched exponential equations are sometimes used for describing the stress relaxation in polymer networks [34, 63-65], thermoplastic elastomers [66], natural rubber [67], or in filled elastomers [68]. In order to take into account the sigmoidal shape of the curves we used the following equation:

$$
E(t)=E_{\infty}+\left(E_{0}-E_{\infty}\right) \exp \left[-\left(t / \tau_{0}\right)^{\mu}\right]
$$

In this equation, $E_{0}$ is the initial value and $E_{\infty}$ is the asymptotic limit. $\tau_{0}$ is the relaxation time and $\mu$ is the stretching exponent $(\mu<1)$. Figure 4 shows that this equation provides a good fit 
for the UP1 data. The initial value $E_{0}$ differs by less than $2 \%$ from the experimental value measured for $t=0$. For the hydroxylated silica filled sample (Si-OH-40-c), Figure 4 reveals an upper limit for the fit. Above $700 \mathrm{~s}, E(t)$ begins to decrease again. It follows that the true asymptotic limit (if any) would be smaller than $E_{\infty}$ determined from the fit with eq 5 . The values of $\mu$ indicated in Figure 4, are slightly larger than the ones (ranging between 0.2 and 0.4 ) reported in the above mentioned publications. The UP2 curves display a clear minimum located around 213, 130 and $105 \mathrm{~s}$ for Si-OH-40-c, Si-OR-40-c and CB-40-c, respectively. This observation suggests the existence of two different regimes. In the short time domain, the elastic modulus decreases (relaxation) toward a plateau. The experimental data can be fitted with eq 5 .

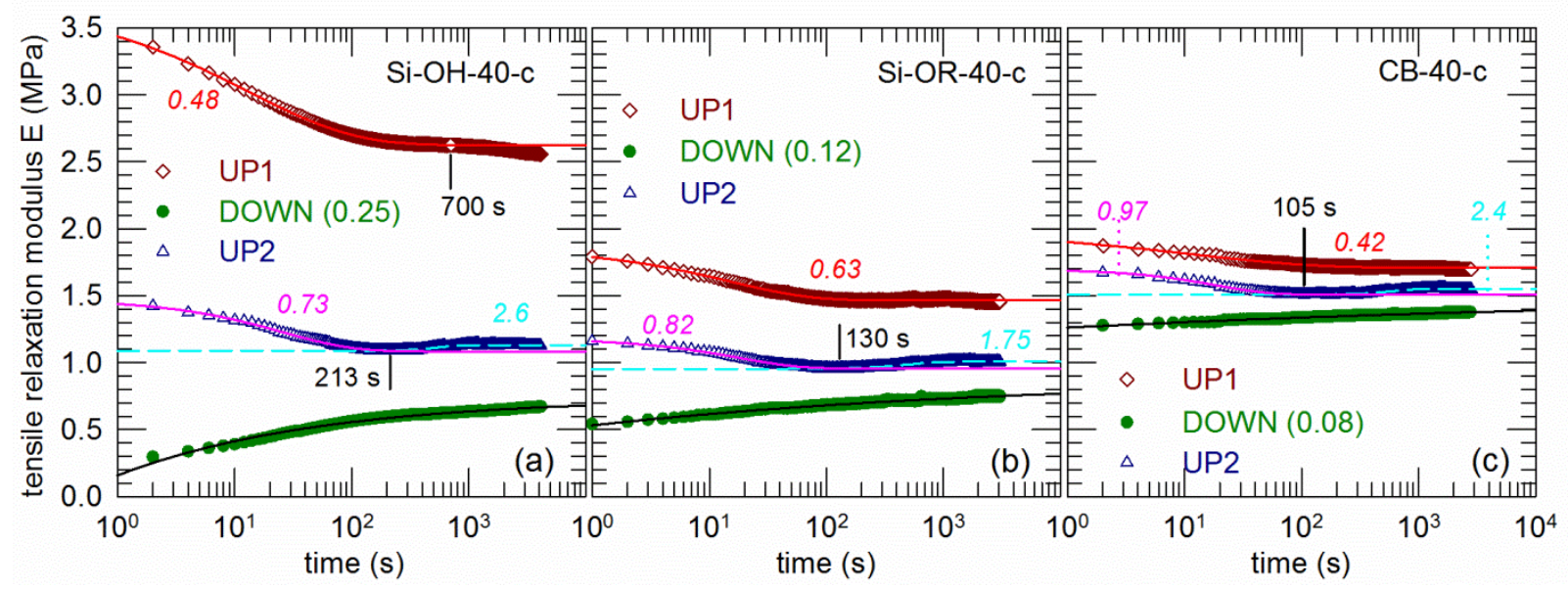

FIGURE 4 Time dependence of the tensile relaxation modulus $\mathrm{E}$ for $\varepsilon=0.2$; the values of $m$ obtained by fitting the DOWN data with eq 4 are indicated in brackets; the values of the exponents $\mu$ deduced from the fit with eq 5 are indicated in italics near the UP curves.

Figure 4 shows that $\mu$ is larger than for the UP1 curves and becomes close to 1 (exponential decrease) for CB-40-c. The characteristic times remain comprised between 13 and $26 \mathrm{~s}$ for the UP1 and UP2 curves. The long time domain is characterized by an increase of $E(t)$ up to a second plateau. It appears that the data can be fitted also with eq 5 but in the present case the 
exponent $\mu$ is larger than 1 (compressed exponential). One may notice that the occurrence of a minimum residual stress at a given time has also been found in aqueous dispersion of laponite following jumps from different shear rates [69].

The fact that the tensile stress relaxation modulus behaves differently at small and at large strains is consistent with observations reported for carbon black filled vulcanized EPDM by GinicMarkovic and coll. [10]. These authors have also shown that such cross-over does not exist in the unfilled matrix which behaves at all strains as the filled matrix at large strains. It follows that, at small strains, the dynamics of the filled elastomer could be governed by the filler particle network involving breaking and recombination of filler particle links. The cross-over between the two regimes could correspond to the strain at which the filler particle network is no longer continuous. A detailed discussion of the physical meaning of these observations goes beyond the scope of the present paper devoted to the power law relaxation and recovery. Further experimental work would be necessary to determine precisely the value of the cross-over strain.

\section{Experimental results obtained for the uncross-linked Si-OH-40-u sample}

Figure 5 shows the curves $E(t)$ obtained for this uncross-linked sample. For strains larger than 0.1 , the curves are well fitted with eq 4 . The values of the exponent $m$ vary between 0.27 and 0.21 (Fig. 5). It may be interesting to mention that the values of the characteristic times $\tau_{0}$ that range between 0.5 and $3 \mathrm{~s}$ are much larger than the ones obtained for the cross-linked sample SiOH-40-c (Table 1). The existence of an asymptotic limit $E_{\infty}$ is not expected in uncross-linked polymers [13]. In the case of a filled polymer, it can be attributed to the network formed by the silica aggregates spanning throughout a "fluid" medium. It follows that the sample Si-OH-40-u could behave formally as a colloidal gel [70]. This point will be discussed in more details in the last part of the paper. As in the case of the cross-linked sample $(\varepsilon=0.2)$, the data obtained for $\varepsilon$ 
$=0.1$ can be fitted by means a stretched exponential (eq 5) up to $700 \mathrm{~s}$. The exponent $\mu(=0.37)$ and the characteristic time $\tau_{0}(=8 \mathrm{~s})$ are slightly smaller than for the cross-linked sample. For times longer than $700 \mathrm{~s}, E(t)$ starts to decrease as observed for the cross-linked sample.

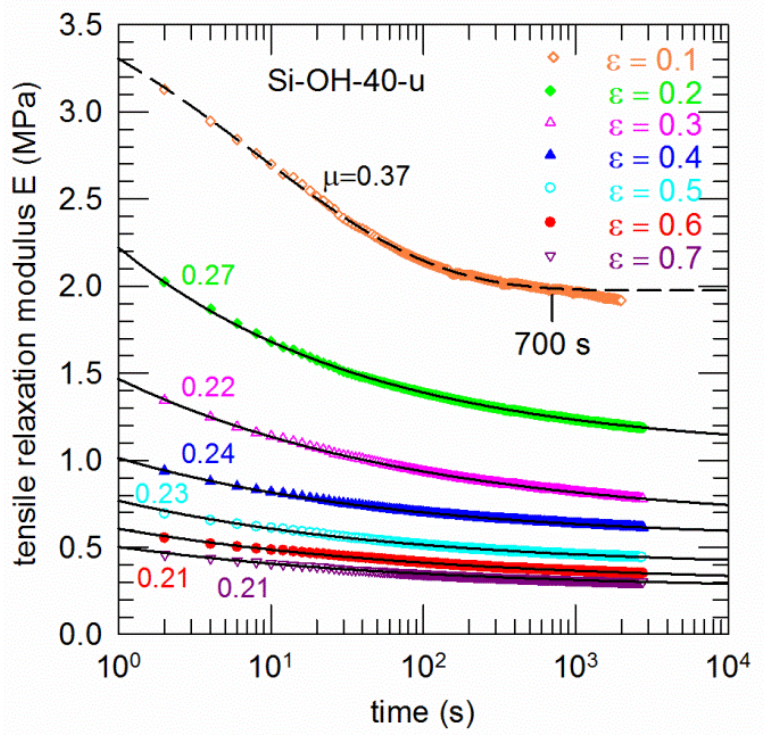

FIGURE 5 Time dependence of the tensile relaxation modulus $\mathrm{E}$ at different strains for the uncross-linked Si-OH-40 sample. Continuous lines correspond to the fit of the data with the power law equation (eq 4); the values of the exponents are indicated near the curves; the dashed line corresponds to the fit with the stretched exponential equation (eq 5) up to $t=700 \mathrm{~s}$.

\section{DISCUSSION}

\section{Physical meaning of the exponent $m$ in the power law equation}

We have shown in the previous paragraph that all $E(t)$ data measured at a given strain $\varepsilon$ larger than 0.2 and reached by increasing the elongation (UP curves) can be fitted by one of the following equations:

- a power law equation (eq 4):

$$
E(t)=E_{\infty}\left[1+k\left(t / \tau_{0}\right)^{-m}\right]
$$


- a logarithmic equation (eq 2): $\mathrm{E}(t)=E(1)[1-K \log (t)]$

In both cases, the derivative which corresponds to the rate of change of the tensile modulus writes as:

$$
|d E(t) / d t| \propto t^{-\alpha}
$$

with $\alpha=m+1$ for the power law equation (eq 4) and $\alpha=1$ for the logarithmic decrease (eq 2).

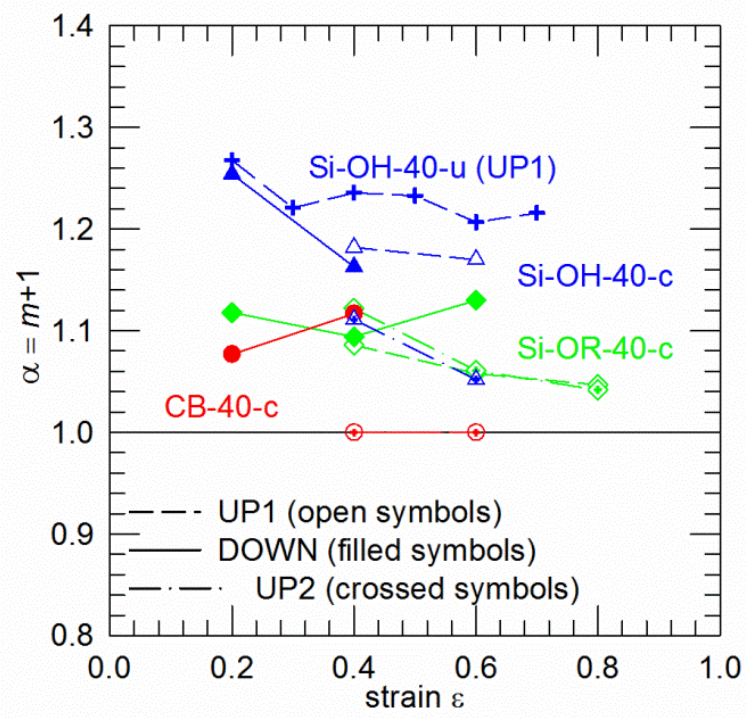

FIGURE 6 Comparison of the values of the exponent $\alpha=m+1$ obtained at different strains for Si-OH-40-u (x), Si-OH-40-c (triangles), Si-OR-40-u (diamonds), CB-40-u (circles). Error bars resulting from the statistical error in the determination of $m$ are smaller than the size of the symbols.

The values of $\alpha=m+1$ are plotted in figure 6 as a function of strain. This figure reveals the following trends:

- $\alpha$ is larger or close to 1.1 for the cross-linked hydroxylated silica samples (Si-OH-c) and larger than 1.2 for the uncross-linked one $(\mathrm{Si}-\mathrm{OH}-\mathrm{u})$

- $\alpha$ is smaller or close to 1.1 for the hydrophobic silica samples ( $\mathrm{Si}-\mathrm{OR}-\mathrm{c})$ and equal to 1 for the CB samples characterized by a $\log (t)$ decrease of the tensile modulus 
- $\alpha$ depends on the initial strain, on the filler-filler and filler-matrix interactions and on the mechanical history of the sample

- $\alpha$ is always strictly larger than 1 during recovery (DOWN).

As a consequence, the exponent $\alpha$ and, therefore, the power law exponent $m=\alpha-1$ (when $\alpha$ is strictly larger than 1) may be considered as a fingerprint of the time-dependent properties of the strained filled elastomers investigated here. Similarly, for soft glassy materials (SGM), the power law exponent $\beta$ characterizing the time-dependent viscoelastic properties is generally labeled as $\beta=x-1$ [36]. The aim of the following discussion is to examine if our model-free exponent $\alpha$ deduced from experimental fits can be assimilated with $x$, the effective noise temperature (or mechanical noise temperature) introduced in the soft glassy rheology (SGR) model [36-38]. The value of $x$ ranges generally between 1.1 and 1.3 for soft glassy materials. The limit $x \rightarrow 1$ corresponds to a glass transition in this model [38, 71]. First of all, it must be reminded that, as mentioned in the introduction, values of $m$ (CT equation) ranging between 0.1 and 0.3 (therefore values of $\alpha$ ranging between 1.1 and 1.3) have been reported also for unfilled elastomers. Tada et al. [72] have shown that the relaxation dynamics in filled rubbers is governed by that of the rubber matrix. Thus, it is likely that $\alpha$ is related to the dynamics of the polymer chains influenced by the filler particles and the strain and not to the filler particles as it would be the case in colloidal glasses below jamming. Under this assumption, the effective noise temperature $x$ could be related to the mobility of structural elements (the polymer chains) relative to the depth of energy wells or cages, in which such elements are trapped. In this case, the glassy behavior $(x=1)$ would be due to the "freezing" of the segmental motion similarly to what occurs at the glass transition temperature of the plain elastomer. In this case, the assumption $\alpha \equiv x$ indicates a glassy state of the elastomer in the strained $(\varepsilon=0.4$ and 0.6$)$ 
carbon black filled sample CB-40-c (UP jumps). It is consistent with the mobility restriction of the polymer chains and the concept of glassy bridges induced by strain in filled elastomers [7375] in the case of strong filler-matrix interaction. On the opposite, none of the silica filled samples exhibits this behavior. As shown in Figure 6, the exponents $\alpha$ are always larger than 1 and never reach the limit $\alpha=1$. The assumption $\alpha \equiv x$ and the observation $\alpha>1$ involve the absence of glassy state in these strained samples in agreement with the observation of the absence of glassy bridges in different series of silica filled elastomers [47-49]. If $\alpha$ actually corresponds to the effective noise temperature $x$, it is expected that a larger $\alpha$ value involves a larger mobility of the polymer chains or chain segments. This is clearly verified in Figure 6 (UP1 relaxations) as $\alpha$ is larger for the uncross-linked matrix than the cross-linked one filled with hydroxylated silica (Si-OH-40-c). For the latter also, $\alpha$ is larger than for the sample filled with hydrophobic silica (Si-OR-40-c). Also, the trend for $\alpha$ to become smaller when the elongation increases (Fig 6) is consistent with a decrease of the chain mobility when being more extended. Similarly, the decrease of the power law exponent with the increase of the elongation of uncrosslinked blends of cis-1,4-polyisoprene (Hevea rubber) and synthetic trans-1,4-polyisoprene blends was attributed by Manzur [76] to a reduction in the mobility of the polymer chains. More generally, a relation between chain mobility and filler matrix interaction involved if $\alpha \equiv x$ is consistent with conclusions reached by Luo et al. [77] who combined NMR and mechanical measurements on silica-silane and carbon black filled styrene-butadiene rubber (SBR). It also agrees with the results reported by Martin and coll. [19] for plasticized EPDM compounds crosslinked with resol, indicating a decrease of $m$ from 0.35 to 0.07 (corresponding to a decrease of $\alpha$ from 1.35 to 1.07 ) with the increase of the cross-link density. In the case of unfilled crosslinked elastomers, the value of $\alpha$ assumed to be an effective noise temperature $x$ would be 
related to the chain mobility decrease near the cross-links. On the contrary, such a relation is in full disagreement with the Curro model [32] in which the exponent $m$ (therefore, $\alpha$ ) increases with the degree of cross-linking. Finally, the strain induced glass state expected if $\alpha \equiv x=1$ for the carbon black filled sample is consistent with our results obtained by the HD-XPCS measurements performed simultaneously with the mechanical ones [58]: in HD-XPCS experiments one studies the decrease of the velocity of the filler particles in the sample during the stress relaxation. We have shown [58] that the velocity of the carbon black particles is small and decreases as a power law of time $t^{-m_{f}}$ and $m_{f}$ close to 2 . For the silica filled samples, the particle velocity is much larger and decreases more slowly $\left(m_{f}\right.$ varies between 1 and 1.2). These results suggest the presence in the latter of viscous domains expected if $\alpha \equiv x>1$ which do not exist for the carbon black filled sample as expected when $\alpha \equiv x=1$.

Furthermore, we expect a more comprehensive description and interpretation of two or multistep viscoelasticity when using SGR based power-laws in the stress response function that is assumed to be separable in time and strain. However, this should be a future task. In such a case we propose starting with the phenomenological Kaye-Bernstein-Kearsley-Zapas (K-BKZ) approach [78]. However, the question whether the SGR model does work in the stress response should then be investigated within the frame of a Doi-Edwards (D-E) model for large-strain viscoelasticity [79]. The D-E model gives insights into the molecular rheology of dense polymers and has been recently successfully applied to morphological and rheological characterization of filled polymer melts and networks in the nonlinear viscoelastic regime [80-82]. Main aspect should then be aimed at possible damage or structural disorder changes seen in differences in upjump and down-jump perturbations of the system [83]. In filled rubber networks these changes may be traced back to stress-induced filler reorganization as investigated by the simultaneous 
XPCS measurements $[58,84]$ and their relation with the dynamics of the cross-linked polymers.

The disorder inherent to SGMs would be captured by assuming that each element of the macroscopic sample has different yield energy [36, 37].

\section{Analysis of the asymptotic limits}

The determination of an asymptotic equilibrium by means of equation 4 allows us to examine the Mullins effect in these systems. To this end, we have plotted on Figures 7 and 8 the values of the stress $\sigma_{0}=\varepsilon E(0)$ measured at $t=0$ shown in Figure 2 and the values of $\sigma_{\infty}=\varepsilon E_{\infty}$ deduced from the fit for the three different fillers. As expected, the second loading curves (UP2) are located between the first ones (UP1) and the unloading curves (DOWN). These figures suggests that the area of the hysteresis loop consisting of UP1 and DOWN curves decreases when going from $\mathrm{Si}-\mathrm{OH}$ to Si-OR and to $\mathrm{CB}$ fillers, i.e., when the filler matrix interaction increases. This qualitative observation agrees with the Mullins effect model [85] based on dissipative friction phenomena due to internal sliding of the macromolecular chains and of the filler particles. It also intuitively agrees with the very different behavior of the filler particle velocity and relaxation investigated by HD-XPCS for Si-OH-40-c and CB-40-c $(\varepsilon=0.60)$ [58]. Within the framework of the SGR model, the decrease of the hysteresis loop area corresponds to smaller values of the effective noise temperature $x$ (assuming that $x$ and $\alpha$ are equivalent) which may be characteristic of a decreasing disorder in the initial samples.

For Si-OR-40-c, the area of the hysteresis loop obtained by considering the asymptotic limits $\sigma_{\infty}$ is significantly reduced (Fig 7b) as compared to Si-OH-40-c (Fig 7a). If the hysteresis observed for $\sigma_{0}$ would be related only to relaxation effects, no hysteresis would be expected for $\sigma_{\infty}$, which is not the case [85]. For CB-40-c (Figure 7c), the logarithmic decrease of the stress with time in the first loading does not yield an asymptotic limit, as expected in a glass. In this case it appears 
that the values of $\sigma_{\infty}(\mathrm{DOWN})$ are very close to that obtained during the second loading (UP2).

The same feature is observed for Si-OR-40-c (Fig 7b) and for Si-OH-40-c $(\varepsilon=0.4)$. Figure 6 also shows that $\alpha$ (i.e., $x$ ) is much smaller (smaller degree of disorder) for the UP2 jump than the UP1 one, at least for $\mathrm{Si}-\mathrm{OH}-40-\mathrm{c}$. It follows that the difference between the first and the second jumps could be explained by a strain induced relief of frozen-in disorder created during the preparation of the sample (curing under pressure at $160^{\circ} \mathrm{C}$ ). This interpretation which would involve some kind of rejuvenation is speculative and requires further theoretical and experimental work.

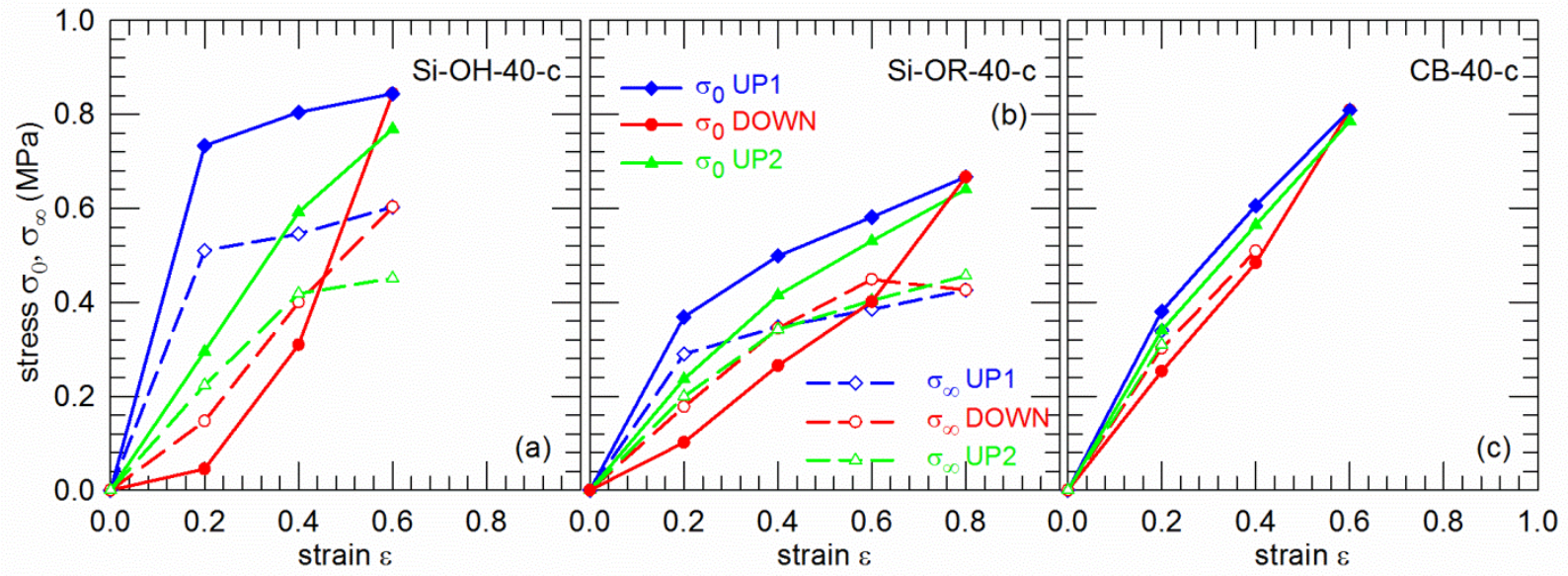

FIGURE 7 Evolution of the initial stress $\sigma_{0}$ and of the asymptotic limit $\sigma_{\infty}$ determined by the power law equation (eq 4): (a) hydroxylated silica, (b) hydrophobic silica and, (c) carbon black fillers [the legend of the 3 figures is indicated in (b)]

The effect of cross-linking on the evolution of $\sigma_{0}$ and $\sigma_{\infty}$ with strain is reported in Figure 8 for the hydroxylated silica filler (Si-OH-40) and compared with Si-OH-40-c (UP1 curves in Fig 7a). For the uncross-linked sample $\mathrm{Si}-\mathrm{OH}-40-\mathrm{u}, \sigma_{0}$ goes through a maximum at $\varepsilon$ close to 0.2 . Such a feature has already been observed for uncross-linked elastomers filled with silica or carbon black. They were shown to be coincident with the appearance of a "butterfly" in the SAXS patterns (at small values of the wave vector $q$ ) and attributed to the breaking and the 
reorganization of the colloidal particle network in the direction of the strain [54]. As discussed in the previous paragraph, the value of $\alpha$ are the largest for Si-OH-40-u which involves a larger chain mobility making possible the reorganization of filler particle network.

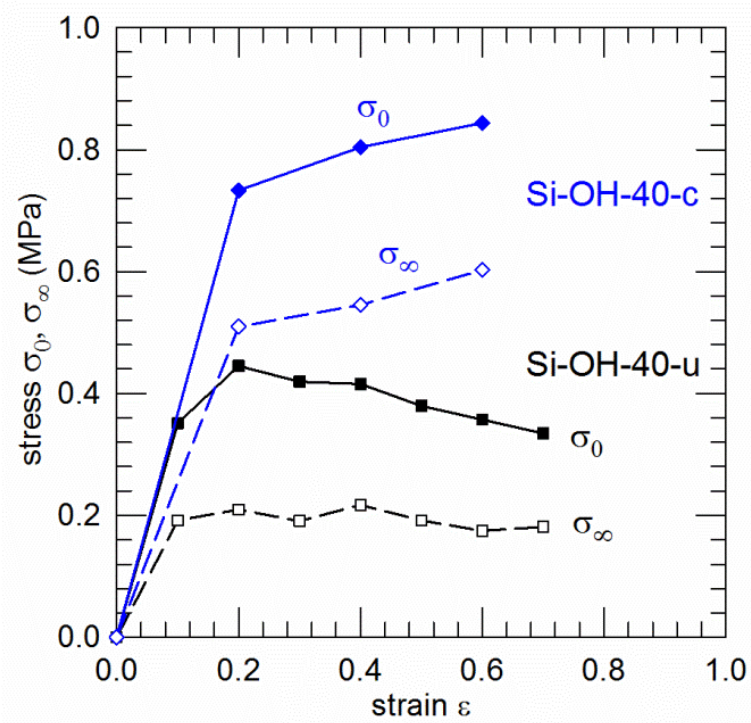

FIGURE 8 Comparison between the evolution with strain of the initial stress $\sigma_{0}$ and the asymptotic limit $\sigma_{\infty}$ (UP1 steps) for cross-linked (Si-OH-40-c) and uncross-linked (Si-OH-40-u) samples

\section{SUMMARY AND CONCLUSION}

The present study was devoted to the analysis of the behavior of the tensile relaxation modulus $E(t)$ at a given strain level reached either by a positive or a negative strain step. The leading parameter of this investigation was the filler-filler or the filler matrix interaction. We have shown that for all silica filled samples strained strictly above 0.2 by positive (UP1 and UP2) or negative (DOWN) jumps, $E(t)$ can be fitted to a power law equation similar to the Chasset-Thirion one. For the carbon black samples characterized by a strong filler-matrix interaction the decrease of $E(t)$ is logarithmic for UP jumps. For DOWN jumps, $E(t)$ follows the same power law equation 
as the silica filled samples. These experimental features indicated that, for all samples, the rate of change of $E(t)$ varies as $t^{-\alpha}$ where $\alpha=m+1$, the logarithmic decrease of $E(t)$ observed only for the carbon black filled sample for UP jump steps corresponding to the limiting case $\alpha=1$. It appeared that the value of the exponent $\alpha$ and, therefore, that of $m$, obtained for a given strain (above a strain threshold) decreases when the filler-matrix interaction increases. Thus, it was suggested that the decrease of the power law exponent $\alpha$ reveals a decrease of the polymer chain mobility. Moreover, the power law relaxation observed in strained filled elastomers describes the local stress relief of a strain quenched disorder as in the case soft glassy materials. It follows that the present series of measurements and the analysis of the data provide experimental clues for soft glassy rheology features in strained filled elastomers. As a consequence, the empirical power law exponent $\alpha$ could be considered as the effective noise temperature $x$ in the SGR model. In the limiting case $x \rightarrow 1$ the system approaches a glass transition which is consistent with the glassy domains reported for strained carbon black filled elastomers. For $x>1$, the increasing agitation of mesoscopic elements permits their relative rearrangements under strain as it is observed for the silica filled samples when the filler matrix interaction decreases which yields an increasing mobility of the polymer chains. We have also shown that the hysteresis effect decreases when $\alpha$ becomes closer to 1 . It follows that the SGR model and the effective noise temperature $x$ could be used for representing the different stress relaxation behaviors observed during loading and unloading experiments aiming to model the Mullins effect. It may be concluded that the experimental work described in the present paper provides strong clues for soft glassy rheology in filled elastomers strained above a given threshold. It is expected that this approach, along with further theoretical refinements, will bring new insights in the mechanical behavior of filled elastomers. 


\section{ACKNOWLEDGEMENTS}

Use of the APS was supported by the DOE, Office of Basis Energy Sciences, under Contract No.

W-31-109-Eng-38. The authors thank René Jurk (IFP Dresden) for the preparation of the samples.

\section{REFERENCES}

[1] T. A. Vilgis, G. Heinrich, M. Klüppel, Reinforcement of Polymer Nanocomposites: Theory, Experiments and Applications (Cambridge University Press, Cambridge, England, 2009).

[2] G. Heinrich, M. Kaliske, A. Lion, S. Reese, Constitutive Models for Rubber VI; Taylor \& Francis Group, London, UK, 2010.

[3] C. M. Roland, Viscoelastic Behavior of Rubbery Materials, Oxford University Press, Oxford, UK (2011); arXiv.org, e-Print Archive, Condensed Matter (2011), 1-46, arXiv:1104.1622v1 [cond-mat.soft].

[4] S. Thomas, R. Stephen, Rubber Nanocomposites: Preparation, Properties and Applications; John Wiley \& Sons (Asia) Pte Ltd Singapore 2010.

[5] S. K. Kumar, R. Krishnamoorti, Annu. Rev. Chem. Biomol. Eng. 2010, 1, 37-58.

[6] J. Oberdisse, W. Pyckhout-Hintzen, E. Straube, In Recent Advances in Polymer Nanocomposites; S. Thomas, G. Zaikov, S. V. Valsaraj, Eds.; Brill NV: Leiden, The Netherlands, 2009; pp 397-438.

[7] G. Allegra, G. Raos, M. Vacatello, Prog. Polym. Sci. 2008, 33, 683-731.

[8] A. Bhattacharya, G. A. Medvedev, J. M. Caruthers, Rubber Chem. Technol. 2011, 84, 296324. 
[9] C. I. MacKenzie, J. Scalan, Polymer 1984, 25, 559-568.

[10] M. Ginic-Markovich, N. K. Dutta, M. Dimopoulos, N. Roy Choudhury, J. G. Matisons, Thermochim. Acta 2000, 357-358, 211-216.

[11] V. G. Geethamma, L. A. Pothen, B. Rhao, N. R. Neelakantan, S. Thomas, J. Appl. Polym. Sci. 2004, 94, 96-104.

[12] J. Sun, H. Li, Y. Song, Q. Zheng, L. He, J. Yu, J. Appl. Polym. Sci. 2009, 112, 3569-3574.

[13] S. Mani, H. H. Winter, M. Silverstein, M. Narkis, Colloid Polym. Sci. 1989, 267, 10021006.

[14] R. Chasset, P. Thirion, In Proceedings of the Conference on Physics of Non-Crystalline Solids; J. A. Prins, Ed.; North-Holland Publishing Co.: Amsterdam, 1965; pp 345-359.

[15] R. A. Dickie, J. D. Ferry, J. Phys. Chem. 1966, 70, 2594-2600.

[16] A. Batra, C. Cohen, L. Archer, Macromolecules 2005, 38, 7174-7180.

[17] D. A. Vega, M. A. Villar, J. L. Alessandrini, E. M. Vallés, Macromolecules 2001, 34, 4591-4596.

[18] L. E. Roth, D. A. Vega, E. M. Vallés, M. A. Villar, Polymer 2004, 45, 5923-5931.

[19] G. Martin, C. Barrès, P. Cassagnau, P. Sonntag, N. Garois, Polymer 2008, 49, 1892-1901.

[20] C. Joubert, A. Michel, L. Choplin, P. Cassagnau, J. Polym. Sci. B 2003, 41, 1779-1790.

[21] K. Aniskevich, O. Starkova, J. Jansons, A. Aniskevich, Mech. Compos. Mater. 2010, 46, 375-386.

[22] S. Mitra, S. Chattopadhyay, A. K. Bhowmick, J. Polym. Res. 2011, 18, 489-497. 
[23] X. Trepat, G. Lenormand, J. J. Fredberg, Soft Matter 2008, 4, 1750-1759.

[24] K. K. Mandadapu, S. Govindjee, M. R. K. Mofrad, J. Biomech. 2008, 41 1467-1478.

[25] P. Kollmannsberger, C. T. Mierke, B. Fabry, Soft Matter 2011, 7, 3127-3132.

[26] M. Balland, N. Desprat, D. Icard, S. Féréol, A. Asnacios, J. Browaeys, S. Hénon, F. Gallet, Phys. Rev. E 2006, 74, 021911.

[27] S. E. Duenwald, R. Vanderby Jr., R. S. Lakes, Ann. Biomed. Eng. 2009, 37, 1131-1140.

[28] S. Hiratsuka, Y. Mizutani, A. Toda, N. Fukushima, K. Kawahara, H. Tokumoto, T. Okajima, Jpn. J. Appl. Phys. 2009, 48, 08JB17.

[29] D. T. N. Chen, Q. Wen, P. A. Janmey, J. C. Crocker, A. G. Yodh, Annu. Rev. Condens. Matter Phys. 2010, 1, 301-322.

[30] A. Jaishankar, G. H. McKinley, Proc. R. Soc. A 2013, 469, 20120284.

[31] M. Rubinstein, S. P. Obukhov, Macromolecules 1993, 26, 1740-1750.

[32] J. G. Curro, P. Pincus, Macromolecules 1983, 16, 559-562.

[33] J. G. Curro, D. S. Pearson, E. Helfand, Macromolecules 1985, 18, 1157-1162.

[34] G. Heinrich, T. A. Vilgis, Macromolecules 1992, 25, 404-407.

[35] G. B. McKenna, R. J. Gaylord, Polymer 1988, 29, 2027-2032.

[36] P. Sollich, F. Lequeux, P. Hébraud, M. E. Cates, Phys. Rev. Lett. 1997, 78, 2020-2023.

[37] P. Sollich, Phys. Rev. E 1998, 58, 738-759.

[38] S. M. Fielding, P. Sollich, M. E. Cates, J. Rheol. 2000, 44, 323-369.

[39] E. Bouchbinder, J. S. Langer, Soft Matter 2013, 9, 8786-8791. 
[40] I. Fuereder, P. Ilg, Phys. Rev. E 2013, 88, 042134.

[41] X. Wang, C. G. Robertson, Phys. Rev. E 2005, 72, 031406.

[42] G. B. McKenna, T. Narita, F. Lequeux, J. Rheol. 2009, 53, 489-516.

[43] G. B. McKenna, J. Res. Natl. Inst. Stand. Technol. 1994, 99, 169-189.

[44] J. Diani, B. Fayolle, P. Gilormini, Eur. Polym. J. 2009, 45, 601-612.

[45] A. F. M. S. Amin, A. Lion, P. Höfer, Z. Angew. Math. Mech. 2010, 90, 347-369.

[46] G. Kraus Angew. Makromol. Chem. 1977, 60-61, 215-48.

[47] N. Jouault, F. Dalmas, S. Said, E. Di Cola, R. Schweins, J. Jestin, F. Boué, Phys. Rev. E 2010, $82,031801$.

[48] C. Chevigny, N. Jouault, F. Dalmas, F. Boué , J. Jestin, J. Polym. Sci., Part B 2011, 49, 781791.

[49] N. Jouault, F. Dalmas, F. Boué, J. Jestin, Polymer 2012, 53, 761-775.

[50] Y. N. Pandey, G. J. Papakonstantopoulos, M. Doxastakis, Macromolecules 2013, 46, 5097 5106.

[51] C.-C. Lin, S. Gam, J. S. Meth, N. Clarke, K. I. Winey, R. J. Composto, Macromolecules 2013, 46, 4502-4509.

[52] T. P. Rieker, M. Hindermann-Bischoff, F. Ehrburger-Dolle, Langmuir 2000, 16, 5588-5592.

[53] F. Ehrburger-Dolle, M. Hindermann-Bischoff, E. Geissler, C. Rochas, F. Bley, F. Livet, Mater. Res. Soc. Symp. 2001, 661, KK7.4.1. 
[54] F. Ehrburger-Dolle, F. Bley, E. Geissler, F. Livet, I. Morfin, C. Rochas, Macromol. Symp. 2003, 200, 157-167.

[55] M. Hindermann-Bischoff, F. Ehrburger-Dolle, Carbon 2001, 39, 375-382.

[56] F. Ehrburger, J. Lahaye, J. Phys. France 1989, 50, 1349-1359.

[57] A. Dorigato, A. Pegoretti, A. Penati, eXPRESS Polym. Lett. 2010, 4, 115-129.

[58] F. Ehrburger-Dolle, I. Morfin, F. Bley, F. Livet, G. Heinrich, S. Richter, L. Piché, M. Sutton, Macromolecules 2012, 45, 8691-8701.

[59] L. J. S. Halloran, A Rheological Study of Stress Relaxation in Elastomers for in situ X-Ray Diffraction Measurements, Master Thesis, McGill University, Montreal, Quebec, Canada, January 20, 2011.

[60] G. Heinrich, F. R. Costa, M. Abdel-Goad, U. Wagenknecht, B. Lauke, V. Härtel, J.

Tschimmel, M. Klüppel, A. L. Svistkov, Kautschuk Gummi Kunststoffe 2005, 58, 163-167.

[61] S. Richter, M. Saphiannikova, K. W. Stöckelhuber, G. Heinrich, Macromol. Symp. 2010, 291-292, 193-201.

[62] S. Richter, H. Kreyenschulte, M. Saphiannikova, T. Götze, G. Heinrich, Macromol. Symp. 2011, 306-307, 141-149.

[63] A. Hotta, S. M. Clarke, E. M. Terentjev, Macromolecules 2002, 35, 271-277.

[64] H. Konyali, Y. Menceloglu, B. Erman, Polymer 2008, 49, 1056-1065.

[65] J. U. Sommer, J. Chem. Phys. 1991, 95, 1316-1317.

[66] A. A. Baeurle, A. Hotta, A. A. Gusev, Polymer 2005, 46, 4344-4354.

[67] S. M. Clarke, F. Elias, E. M. Terentjev, Eur. Phys. J. E 2000, 2, 335-341. 
[68] A. P. Meera, S. Said, Y. Grohens, A. S. Luyt, S. Thomas, Ind. Eng. Chem. Res. 2009, 48, 3410-3416.

[69] A. S. Negi, C. O. Osuji, J. Rheol. 2010, 54, 943-958.

[70] P. J. Lu, D. Weitz, Ann. Rev. Conden. Matter Phys. 2013, 4, 217-233.

[71] R. Bandyopadhyay, D. Liang, J. L. Harden, R. L. Leheny, Solid State Commun. 2006, 139, $589-598$.

[72] T. Tada, K. Urayama, T. Mabuchi, K. Muraoka, T. Takigawa, J. Polym. Sci.: Part B:

Polym. Phys. 2010, 48, 1380-1387.

[73] S. Merabia, P. Sotta, D. R. Long, J. Polym. Sci.: Part B: Polym. Phys. 2010, 48, 1495-1508.

[74] A. Papon, S. Merabia, L. Guy, F. Lequeux, H. Montes, P. Sotta, D. R. Long, Macromolecules 2012, 45, 2891-2904.

[75] A. Papon, H. Montes, F. Lequeux, J. Oberdisse, K. Saalwächter, L. Guy, Soft Matter 2012, 8, 4090-4096.

[76] A. Manzur, Rev. Mex. Fís. 1991, 37 Supl. 1, 585-592.

[77] H. Luo, M. Klüppel, H. Schneider, Macromolecules 2004, 37, 8000-8009.

[78] V. Rouiller, G. B. McKenna, Soc. Plast. Eng., ANTEC 98, 1998, II, 2138-2142.

[79] M. Doi, S. F. Edwards, The theory of polymer dynamics; Oxford University Press, Oxford 1988.

[80] A. I. Leonov, J. Rheol. 1990, 34, 1039-1068.

[81] S. Richter, M. Saphiannikova, D. Jehnichen, M. Bierdel, G. Heinrich, eXPRESS Polym. Lett., 2009, 3, 753-768. 
[82] M. Rendek, A. Lion, Z. Angew. Math. Mech. 2010, 90, 436 - 458.

[83] G. B. McKenna, T. Narita, F. Lequeux, J. Rheol. 2013, 57, 1803-1818.

[84] F. Ehrburger-Dolle, I. Morfin, F. Bley, F. Livet, G. Heinrich, S. Richter, L. Piché, M.

Sutton, In CP1092 Synchrotron Radiation in Materials Science: 6th International Conference;

Magalhaes-Paniago, R., Ed.; American Institute of Physics: Melville, NY, 2009; pp 29-33.

[85] S. Cantournet, R. Desmorat, J. Besson, Int. J. Solids Struct. 2009, 46, 2255-2264. 